\title{
Adjuvant chemotherapy and survival among patients 70 years of age and younger with node-negative breast cancer and the 21-gene recurrence score of 26-30
}

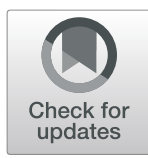

Seho Park ${ }^{1,2}$, Yunan Han ${ }^{1,3}$, Ying Liư ${ }^{1}$, Adetunji T. Toriola', Lindsay L. Peterson ${ }^{4}$, Graham A. Colditz', Seung $\|$ Kim² $^{2}$ Young Up Cho ${ }^{2}$, Byeong-Woo Park² and Yikyung Park ${ }^{1 *}$

\begin{abstract}
Background: The benefits of chemotherapy in node-negative, hormone receptor-positive, and human epidermal growth factor receptor 2 (HER2)-negative breast cancer patients with the 21-gene recurrence score (RS) of 18-30, particularly those with RS 26-30, are not known.

Methods: Using the Surveillance, Epidemiology, and End Results (SEER) data, we retrospectively identified 29,137 breast cancer patients with the 21-gene RS of 18-30 diagnosed between 2004 and 2015. Mortality risks according to the RS and chemotherapy use were compared by the Kaplan-Meier method and Cox's proportional hazards model.

Results: Among the breast cancer patients with the RS 18-30, 21\% of them had RS 26-30. Compared to breast cancer patients with RS 18-25, patients with RS 26-30 had more aggressive tumor characteristics and chemotherapy use and increased risk of breast cancer-specific mortality and overall mortality. In breast cancer patients who were aged $\leq 70$ years and had RS of 26-30, chemotherapy administration was associated with a 32\% lower risk of breast cancer-specific mortality (hazard ratio [HR], 0.68; 95\% confidence interval [Cl], 0.47-0.99) and a $42 \%$ lower risk of overall mortality (HR, $0.58 ; 95 \% \mathrm{Cl}, 0.44-0.76)$. Survival benefits were most pronounced in breast cancer patients who were younger or had grade III tumor.

Conclusions: The 21-gene RS of 18-30 showed heterogeneous outcomes, and the RS 26-30 was a significant prognostic factor for an increased risk of mortality. Adjuvant chemotherapy could improve the survival of nodenegative, hormone receptor-positive, and HER2-negative breast cancer patients with the 21-gene RS 26-30 and should be considered for patients, especially younger patients or patients with high-grade tumors.
\end{abstract}

Keywords: Breast cancer, Chemotherapy, Survival, 21-gene recurrence score

\section{Introduction}

Systemic chemotherapy reduces the risk of recurrence and mortality in estrogen receptor (ER) and/or progesterone receptor (PR)-positive breast cancer patients irrespective of lymph node status [1]. However, the benefits of chemotherapy are not experienced by all breast cancer patients, leading to a need to identify patients who

\footnotetext{
* Correspondence: yikyungpark@wustl.edu

${ }^{1}$ Division of Public Health Sciences, Department of Surgery, Washington University School of Medicine, 660 South Euclid Avenue, Campus Box 8100, St. Louis, MO 63110, USA

Full list of author information is available at the end of the article
}

are more likely to benefit from adjuvant chemotherapy. The 21-gene recurrence score (RS) assay (Oncotype DX'; Genomic Health, Inc., Redwood City, CA) available since 2004 is one of the promising tools to guide treatment decisions in hormone receptor-positive and human epidermal growth factor receptor 2 (HER2)-negative breast cancer patients [2,3]. Based on the 21-gene RS assay, patients are classified into high (score $\geq 31$ ), intermediate (score 18-30), and low (score $\leq 17)$ risk. Studies found that patients in the high-risk group of RS $\geq 31$ had a significant benefit from chemotherapy [4], while patients with the RS $<11$ or $11-25$ who were treated with

(c) The Author(s). 2019 Open Access This article is distributed under the terms of the Creative Commons Attribution 4.0 International License (http://creativecommons.org/licenses/by/4.0/), which permits unrestricted use, distribution, and 
Table 1 Demographic and clinicopathological characteristics of study population

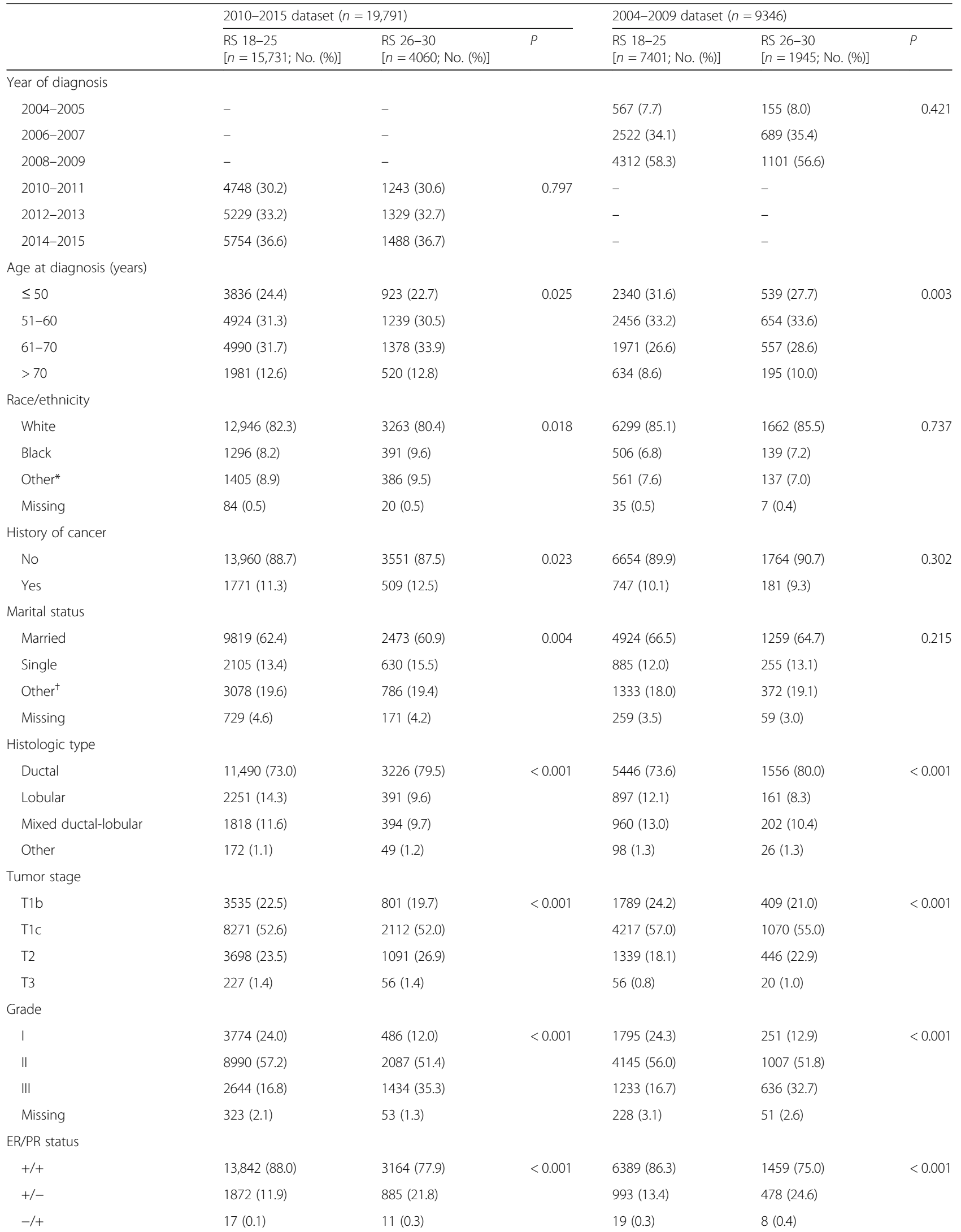


Table 1 Demographic and clinicopathological characteristics of study population (Continued)

\begin{tabular}{|c|c|c|c|c|c|c|}
\hline & \multicolumn{3}{|c|}{ 2010-2015 dataset $(n=19,791)$} & \multicolumn{3}{|c|}{ 2004-2009 dataset $(n=9346)$} \\
\hline & $\begin{array}{l}\text { RS } 18-25 \\
{[n=15,731 ; \text { No. (\%)] }}\end{array}$ & $\begin{array}{l}\text { RS 26-30 } \\
{[n=4060 ; \text { No. }(\%)]}\end{array}$ & $P$ & $\begin{array}{l}\text { RS } 18-25 \\
{[n=7401 ; \text { No. }(\%)]}\end{array}$ & $\begin{array}{l}\text { RS } 26-30 \\
{[n=1945 ; \text { No. }(\%)]}\end{array}$ & $P$ \\
\hline \multicolumn{7}{|l|}{ Type of surgery } \\
\hline Breast-conservation surgery & $10,806(68.7)$ & $2747(67.7)$ & 0.207 & $5199(70.3)$ & $1326(68.2)$ & 0.076 \\
\hline Mastectomy & $4925(31.3)$ & $1313(32.3)$ & & $2202(29.8)$ & $619(31.8)$ & \\
\hline \multicolumn{7}{|l|}{ Radiation therapy } \\
\hline No or unknown & $6268(39.8)$ & $1834(45.2)$ & $<0.001$ & $3038(41.1)$ & $853(43.9)$ & 0.025 \\
\hline Yes & $9463(60.2)$ & $2226(54.8)$ & & $4363(59.0)$ & $1092(56.1)$ & \\
\hline \multicolumn{7}{|l|}{ Chemotherapy } \\
\hline No or unknown & $11,832(75.2)$ & $1807(44.5)$ & $<0.001$ & $5044(68.2)$ & $865(44.5)$ & $<0.001$ \\
\hline Yes & $3899(24.8)$ & $2253(55.5)$ & & $2357(31.9)$ & $1080(55.5)$ & \\
\hline
\end{tabular}

$R S$ recurrence score, No. number, $E R$ estrogen receptor, $P R$ progesterone receptor

${ }^{*}$ Other race includes American Indian, Alaska Native, and Asian or Pacific Islander

${ }^{\dagger}$ Other marital status includes separated, divorced, and widowed categories

endocrine therapy alone had excellent survival outcomes without chemotherapy in the Trial Assigning Individualized Options for Treatment (TAILORx) trial $[5,6]$.

Although patients with the intermediate 21-gene RS of $18-30$ seemed to have no clinical benefits from chemotherapy in previous studies $[3,7,8]$, young women of $\leq 50$ years old with the RS 16-25 who received chemotherapy had lower distant recurrence rates than women who did not receive chemotherapy [6]. A recent study using the National Cancer Data Base (NCDB) also found that combination of chemotherapy and hormone therapy was associated with a lower risk of overall mortality in node-negative breast cancer patients with the RS $18-25$ as well as those with the RS 26-30 than patients who did not receive chemotherapy [9]. However, this study included older patients ( $>70$ years of age) and those with very small tumors of $\leq 0.5 \mathrm{~cm}$, or favorable histologies, for whom the National Comprehensive Cancer Network (NCCN) clinical practice guidelines for breast cancer do not recommend multigene assays [10]; therefore, more specified analyses focusing on an intermediate RS group are required.

Given that the uncertainty of chemotherapy benefits remains in intermediate-risk patients, especially with the RS of 26-30 in hormone receptor-positive, HER2-negative, and node-negative breast cancers, we investigated whether adjuvant chemotherapy was associated with breast cancerspecific mortality and overall mortality in breast cancer patients with the RS $18-30$, particularly among those aged $\leq 70$ years with the RS $26-30$.

\section{Patients and methods}

The Surveillance, Epidemiology, and End Results (SEER) database

Since 1973, the SEER has collected cancer incidence and survival data, including patient demographics; tumor characteristics of primary site, grade, and stage at diagnosis; and first course of treatments from 18 population-based cancer registries. The SEER registry has also collected ER and PR status of breast cancer since 1990 and HER2 status since 2010. All breast cancers diagnosed between 2004 and 2015 (excluding cases reported by the Alaska Native Tumor Registry) were linked to the 21-gene RS data provided by the Genomic Health Clinical Laboratory [11]. To maximize the use of Oncotype DX data and have longer follow-up time, we included all 21-gene RS data since its first collection in 2004. We obtained permission to use the SEER custom data with additional radiation therapy and chemotherapy information and the 21-gene RS data.

\section{Study population}

We identified 111,635 breast cancer cases with the 21gene RS in the SEER 18 database from 2004 to 2015. We first excluded male breast cancer patients and cases identified at death/autopsy or through death certificate only (Additional file 1: Figure S1). Based on the NCCN guidelines for use of multigene assay [10], we also excluded cases with at least one of the following conditions: tumors with non-epithelial origin including sarcoma (International Classification of Diseases for Oncology, 3rd Edition, ICD-O-3 > 8800), tubular (8211), mucinous (8453, 8480, and 8481), and papillary (8050, 8260, and 8503) subtypes; T0-T1a or T4 stage; lymph node-positive $(\geq \mathrm{N} 1 \mathrm{mi})$; distant metastasis (M1) or unknown stage at diagnosis; ER/PR-negative or unknown ER/PR status; or cases whose 21-gene RS results were obtained $>12$ months after breast cancer diagnosis. In addition, we excluded breast cancer cases with the 21-gene RS $<18$ (low-risk category) or $>30$ (high-risk category). Because HER2 status was available from 2010, we divided the study population into two groups based on time, 2004-2009 and 2010-2015. In the 2010-2015 dataset, we further excluded breast cancer cases whose HER2 status was 
positive, borderline, or unknown. The final analytic cohorts consisted of 9346 breast cancer patients in 20042009 dataset and 19,791 patients in 2010-2015 dataset. Because of our de-identified data release from the SEER program and study completion in accordance with the SEER data-use agreement, this study was exempted from the Institutional Review Board approval.

\section{Clinicopathological information and outcomes}

The SEER data provided patient's demographics, such as age at cancer diagnosis; race/ethnicity; marital status; history of other cancer; tumor characteristics including morphology, stage, grade, and ER/PR status; and first course of treatment-type of surgery (breast-conservation surgery and mastectomy), radiation therapy (yes and no/unknown), and chemotherapy (yes and no/unknown). We defined breast cancer histology: ductal (ICD-O-3, 8500), lobular (8520), mixed ductal-lobular (8522, 8523, and 8524), and other type.

Underlying causes of death and durations of survival in the SEER registries were ascertained through linkage to the state death certificates and the National Death Index from the National Center for Health Statistics [12]. We used these variables recorded in the SEER database for calculating breast cancer-specific survival and overall survival.

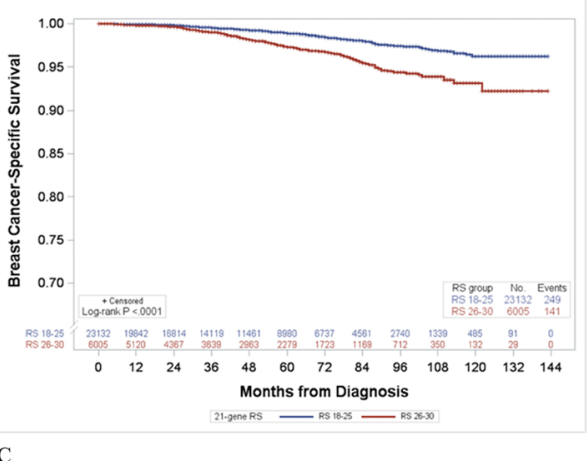

C

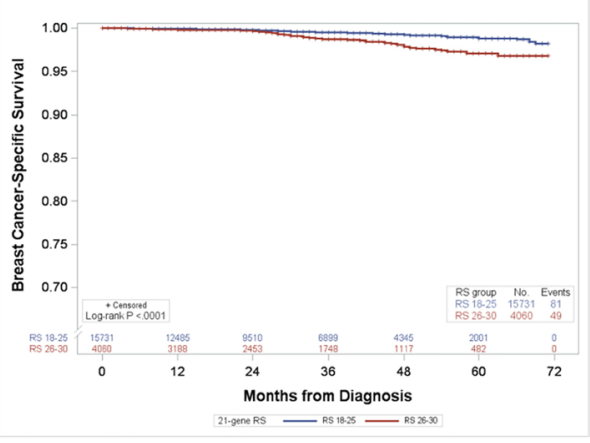

E

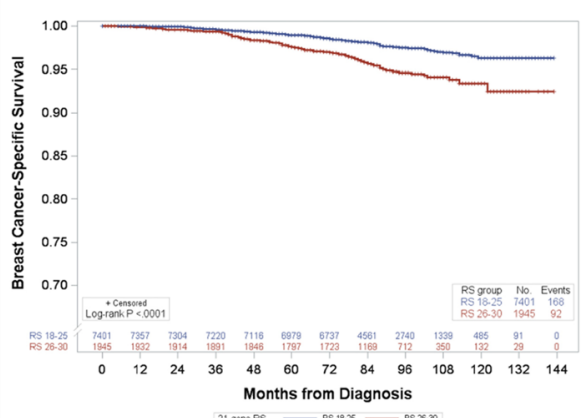

B

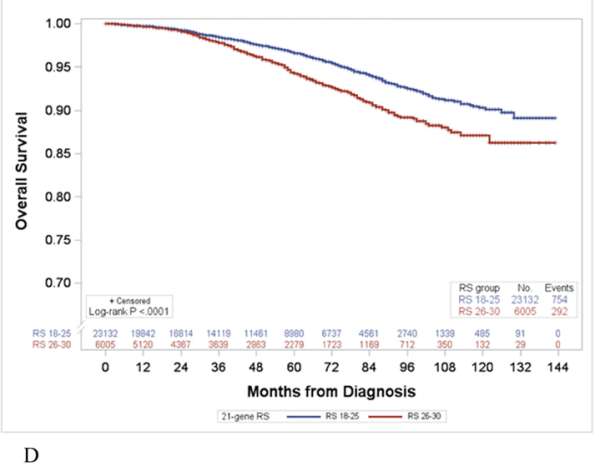

$\mathrm{D}$
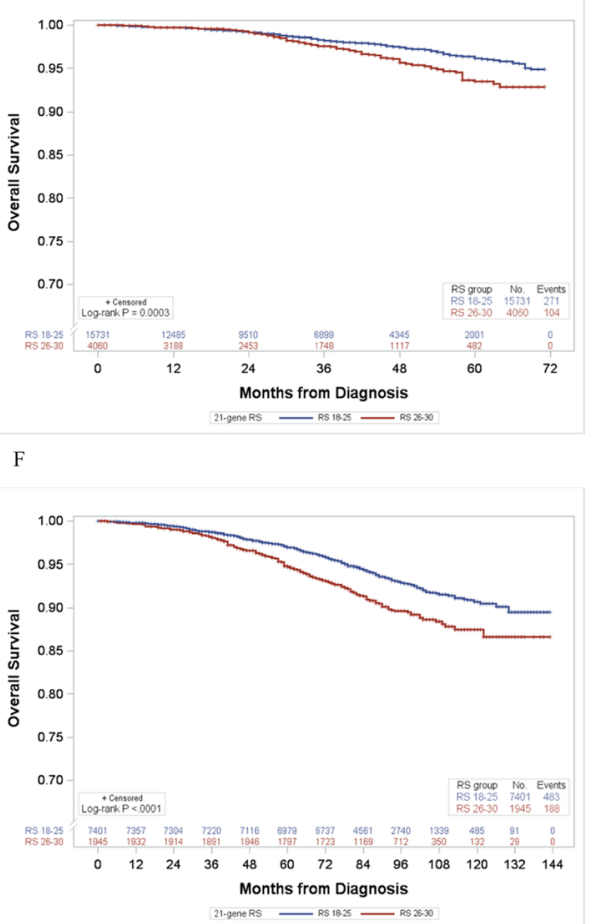

Fig. 1 Breast cancer-specific and overall survival curves according to the 21-gene recurrence score (RS). a Breast cancer-specific survival and $\mathbf{b}$ overall survival in 2004-2015 ( $n=29,137)$. c Breast cancer-specific survival and d overall survival in 2010-2015 $(n=19,791)$. e Breast cancer-specific survival and f overall survival in 2004-2009 $(n=9346)$ 


\section{Statistical analysis}

We conducted analyses in the 2004-2009 and 2010-2015 (HER2-negative case only) dataset, separately, and the 2004-2015 combined datasets. Based on cutoffs in the TAILORx trial [13], we categorized patients into the 21gene RS 18-25 and 26-30 groups and compared demographic and clinicopathological characteristics: differences in means for continuous variables and percentages for categorical variables between two groups were tested using $t$ test and chi-square test, respectively. Survival curves were plotted by the Kaplan-Meier method and differences in survival time were calculated by the log-rank test.

Person-months of follow-up were calculated from the date of breast cancer diagnosis to date of death, known last follow-up, or follow-up end date (December 31, 2015), whichever occurred first. Hazard ratios (HRs) and 95\% confidence intervals (CIs) for breast cancer-specific and all-cause mortality were estimated by the Cox's proportional hazards model with person-months as time scale. A missing category was created for race/ethnicity $(0.5 \%)$, marital status $(4.2 \%)$, and grade $(2.3 \%)$. We examined age-adjusted and multivariate models adjusting for age at diagnosis, calendar year of breast cancer diagnosis (2-year interval), race/ethnicity (White, Black, and others), marital status (married and single/other), history of cancer (yes and no), histologic type (ductal, lobular, and mixed ductal-lobular/other), tumor stage (T1b, T1c, and T2-3), grade (I, II, and III), and ER/PR status $(E R+/ P R+$, $\mathrm{ER}+/ \mathrm{PR}-$, and $\mathrm{ER}-/ \mathrm{PR}+$ ), surgery (breast-conservation surgery and mastectomy), radiation therapy (yes, and no/ unknown), and chemotherapy (yes and no/unknown). We also calculated the mortality risk by chemotherapy status in patients who had the 21-gene RS $26-30$ and were $\leq 70$ years old. We conducted sensitivity analyses in the 20102015 dataset by including breast cancer cases with HER2 positive, borderline, or unknown.

Breast cancer cases were generated and exported using SEER*Stat software version 8.3.5 (https://seer.cancer.gov/ seerstat). All statistical analyses were performed using

Table 2 Hazard ratios (HR) for breast cancer-specific and overall mortality by the 21-gene RS

\begin{tabular}{|c|c|c|c|c|}
\hline & \multicolumn{2}{|c|}{ Breast cancer-specific mortality } & \multicolumn{2}{|c|}{ Overall mortality } \\
\hline & $\begin{array}{l}\text { RS 18-25 } \\
\text { (Reference) }\end{array}$ & $\begin{array}{l}\text { RS 26-30 } \\
{[H R(95 \% C l)]}\end{array}$ & $\begin{array}{l}\text { RS } 18-25 \\
\text { (Reference) }\end{array}$ & $\begin{array}{l}\text { RS 26-30 } \\
{[\mathrm{HR}(95 \% \mathrm{Cl})]}\end{array}$ \\
\hline \multicolumn{5}{|l|}{ 2004-2015 dataset $(n=29,137)$} \\
\hline No. of events & 249 & 141 & 754 & 292 \\
\hline Age-adjusted model & 1.00 & $2.17(1.76$ to 2.67$)$ & 1.00 & $1.43(1.25$ to 1.64$)$ \\
\hline Age- and clinicopathological factor-adjusted model & 1.00 & $1.81(1.46$ to 2.24$)$ & 1.00 & $1.32(1.15$ to 1.52$)$ \\
\hline Age- and clinicopathological and treatment factor-adjusted model & 1.00 & $1.81(1.46$ to 2.26$)$ & 1.00 & $1.37(1.19$ to 1.58$)$ \\
\hline $\begin{array}{l}\text { Continuous RS in age- and clinicopathological and treatment } \\
\text { factor-adjusted model* }\end{array}$ & \multicolumn{2}{|c|}{1.09 (1.06 to 1.12$) / 1$-unit $\mathrm{RS}$} & \multicolumn{2}{|c|}{1.05 (1.03 to 1.07$) / 1-$ unit RS } \\
\hline \multicolumn{5}{|l|}{ 2010-2015 dataset $(n=19,791)$} \\
\hline No. of events & 81 & 49 & 271 & 104 \\
\hline Age-adjusted model & 1.00 & 2.35 (1.65 to 3.36$)$ & 1.00 & $1.46(1.16$ to 1.83$)$ \\
\hline Age- and clinicopathological factor-adjusted model & 1.00 & $1.84(1.27$ to 2.67$)$ & 1.00 & $1.27(1.01$ to 1.61$)$ \\
\hline Age- and clinicopathological and treatment factor-adjusted model & 1.00 & $1.83(1.25$ to 2.69$)$ & 1.00 & $1.28(1.00$ to 1.63$)$ \\
\hline $\begin{array}{l}\text { Continuous RS in age- and clinicopathological and treatment } \\
\text { factor-adjusted model* }\end{array}$ & \multicolumn{2}{|c|}{$1.12(1.06$ to 1.18$) / 1$-unit $\mathrm{RS}$} & \multicolumn{2}{|c|}{1.05 (1.02 to 1.08)/1-unit RS } \\
\hline \multicolumn{5}{|l|}{ 2004-2009 dataset $(n=9346)$} \\
\hline No. of events & 168 & 92 & 483 & 188 \\
\hline Age-adjusted model & 1.00 & 2.08 (1.61 to 2.68$)$ & 1.00 & $1.42(1.20$ to 1.68$)$ \\
\hline Age- and clinicopathological factor-adjusted model & 1.00 & 1.79 (1.38 to 2.33$)$ & 1.00 & $1.35(1.14$ to 1.61$)$ \\
\hline Age- and clinicopathological and treatment factor-adjusted model & 1.00 & 1.80 (1.38 to 2.36$)$ & 1.00 & $1.41(1.18$ to 1.69$)$ \\
\hline $\begin{array}{l}\text { Continuous RS in age- and clinicopathological and treatment } \\
\text { factor-adjusted model* }\end{array}$ & \multicolumn{2}{|c|}{1.07 (1.04 to 1.11$) / 1$-unit RS } & \multicolumn{2}{|c|}{1.05 (1.03 to 1.07$) / 1$-unit RS } \\
\hline \multicolumn{5}{|c|}{$\begin{array}{l}\text { Age at diagnosis is used by categorization into } \leq 50,51-60,61-70 \text {, and }>70 \text { years. Adjusted clinicopathological factors are year of diagnosis (2004-2005, 2006- } \\
2007,2008-2009 \text { in 2004-2009 dataset and 2010-2011, 2012-2013, and 2014-2015 in 2010-2015 dataset), race/ethnicity (White, Black, and other), history of } \\
\text { cancer (no and yes), marital status (married and single/other), histologic type (ductal, lobular, and mixed ductal-lobular/other), tumor stage (T1b, T1C, and T2-3), } \\
\text { grade (I, II, III, and missing), and ER/PR status (both ER/PR-positive and either ER/PR-positive). Treatment factors are type of surgery (breast-conservation surgery } \\
\text { and mastectomy), radiation therapy (no/unknown and yes), and chemotherapy (no/unknown and yes) } \\
\text { RS recurrence score, HR hazard ratio, Cl confidence interval, No. number }\end{array}$} \\
\hline
\end{tabular}


SAS software version 9.4 (SAS Institute Inc., Cary, NC), and a $P$ value $<0.05$ was considered statistically significant for 2-sided tests.

\section{Results}

Mortality risk according to the 21-gene RS

There were 19,791 breast cancer patients in the 20102015 (mean follow-up 32 months) and 9346 patients in the 2004-2009 dataset (mean follow-up 89 months; Table 1). In both datasets, $21 \%$ of patients had the 21 gene RS of 26-30. The distribution of the 21-gene RS was not different between the 2010-2015 and 2004-2009 datasets (Additional file 2: Figure S2). Compared to patients with the RS 18-25, those with the 21-gene RS 2630 were more likely to be older, non-White, not married, have ductal histology, advanced tumor stage, higher grade tumor, or frequent loss of ER or PR, and receive chemotherapy, but no/unknown radiation therapy.

Patients with the RS 26-30 in the 2004-2015 dataset had significantly worse breast cancer-specific survival and overall survival compared to those with the RS 18-25 (Fig. 1a, b). When the survival was examined in the 20102015 and 2004-2009 dataset, separately, we found similar results: poor survival in women with the RS 26-30 in each dataset (Fig. 1c-f). Compared to patients with the RS 18-25, those with the RS 26-30 had an $81 \%$ increased risk of breast cancer-specific mortality (HR, 1.81; 95\% CI, 1.46-2.26) and a 37\% increased risk of overall mortality (HR,1.37; 95\% CI, 1.19-1.58; Table 2) after adjusting for potential confounders, including age at diagnosis, clinicopathological characteristics, and treatments. Similar results were consistently observed when we analyzed the 2010-2015 and 2004-2009 dataset, separately.

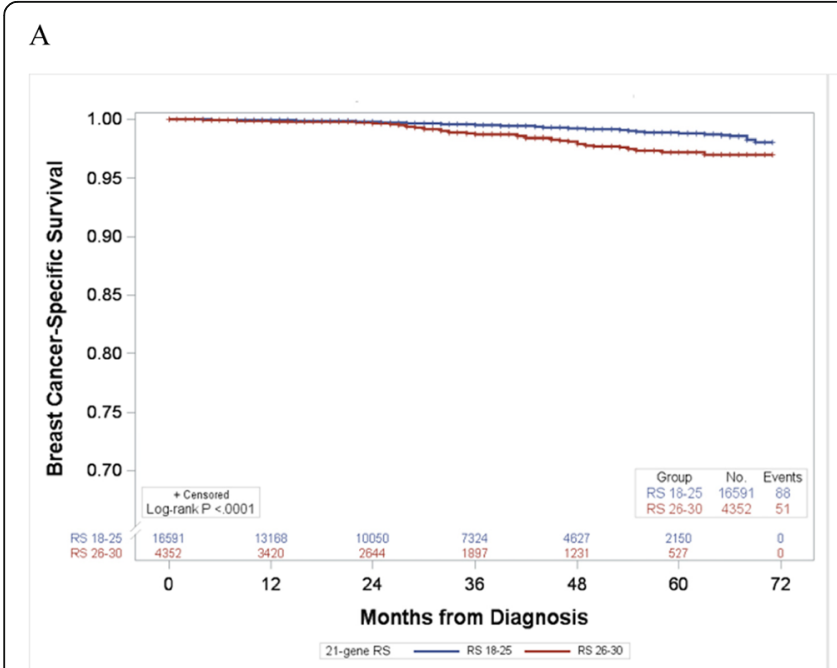

C

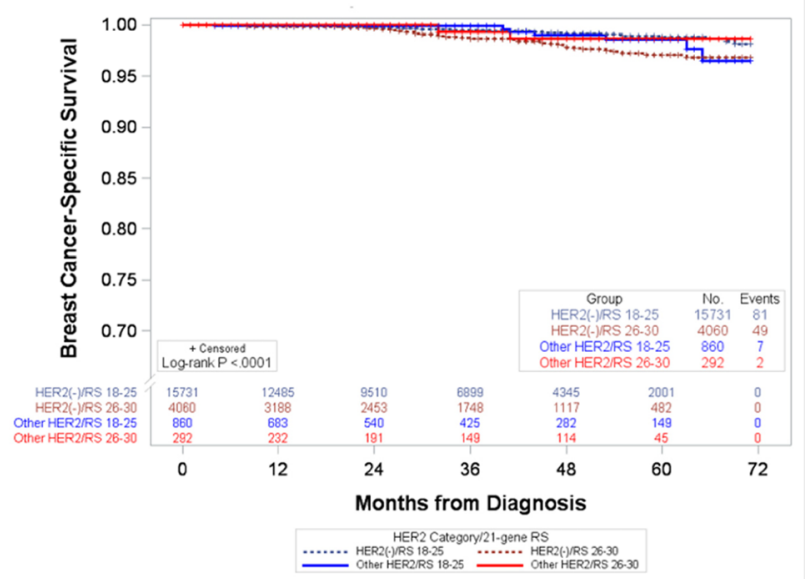

B

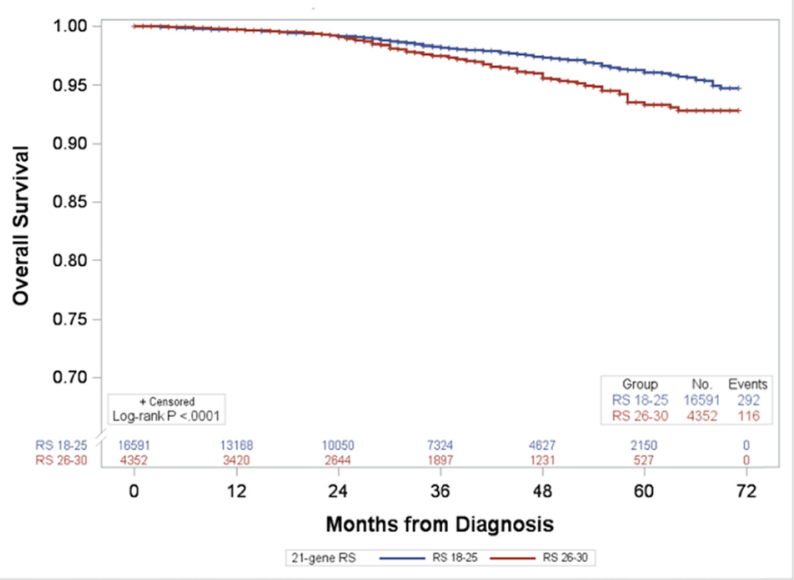

$\mathrm{D}$

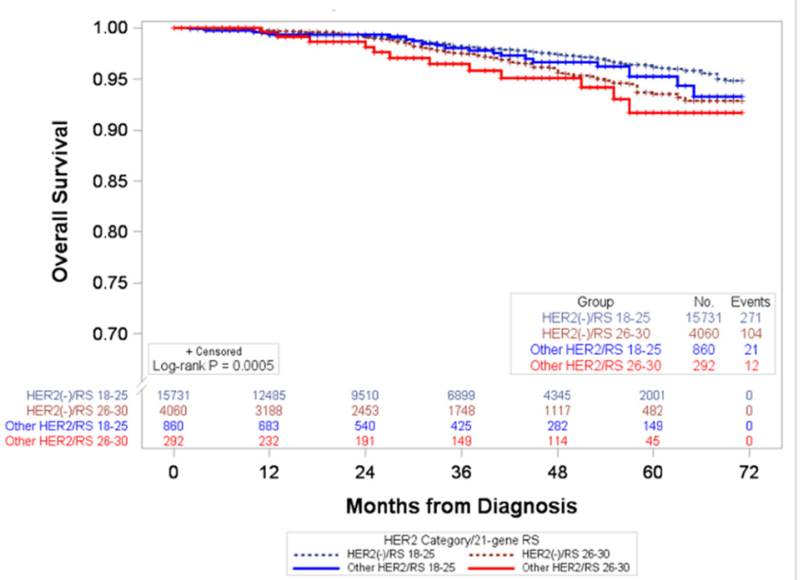

Fig. 2 Breast cancer-specific and overall survival curves according to the 21-gene RS in sensitivity analyses that included breast cancer with HER2positive, borderline, and unknown status in 2010-2015 ( $n=20,943)$. a Breast cancer-specific survival and b overall survival according to RS groups. c Breast cancer-specific survival and $\mathbf{d}$ overall survival according to RS group by HER2 status 
In sensitivity analyses that included breast cancer with HER2-positive, borderline, or unknown status in 20102015 dataset ( $n=1152,5.5 \%$ of total breast cancer cases), similar results were found: patients with the RS 26-30 had poor survival than those with the RS 18-25 (Fig. 2). Also, after further adjusting for HER2 status in multivariate models, there was a $78 \%$ and $31 \%$ increased risk of breast cancer-specific and overall mortality, respectively, in patients with the RS 26-30 compared to those with the RS 18-25 (Additional file 3; Table S1).

\section{Chemotherapy and survival of patients with the RS 26-30 and age $\leq 70$ years}

Among 5290 breast cancer patients aged $\leq 70$ years with the RS 26-30, 3130 (59\%) patients received adjuvant chemotherapy. Patients who received chemotherapy were more likely to be younger and have advanced tumor stage, grade III, and also receiving radiation therapy than those who did not (Additional file 4: Table S2). In the 2004-2015 dataset, breast cancer-specific survival did not significantly differ by chemotherapy use (log-rank $P=0.089$; Fig. 3a). On the other hand, overall survival rate was significantly higher in patients who received chemotherapy than in those who did not (log-rank $P<0.001$; Fig. 3b). When breast cancerspecific survival by chemotherapy use was examined in subgroups of age ( $\leq 56$ and $57-70$ years old: mean age $=56$ years $)$ and grade (I-II and III), younger patients of $\leq 56$ years (log-rank $P=0.046$; Fig. $4 \mathrm{a})$ and patients with grade III tumor (log-rank $P=0.003$; Fig. 4g) tended to have better survival when they received chemotherapy than those who did not. Overall survival was consistently higher in patients who received chemotherapy regardless of their age and grade (Fig. 4). Similar results were found in analyses using the 2004-2009 and 2010-2015 data, separately (data not shown).

In multivariate Cox's hazard models, adjuvant chemotherapy was associated with significantly decreased risks of breast cancer-specific mortality ( $\mathrm{HR}, 0.68 ; 95 \% \mathrm{CI}$, $0.47-0.99$ ) and overall mortality (HR, 0.58; 95\% CI, 0.44-0.76) after adjusting for demographic, clinicopathological, and treatment factors in the 2004-2015 dataset (Table 3). Similar, but statistically not significant, associations were observed in analyses of the 2010-2015 and 2004-2009 datasets, separately.

\section{Discussion}

In this study, we found that the 21-gene RS was associated with an increased risk of breast cancer-specific and overall mortality in breast cancer patients who had the RS18-30. In addition, our study suggested that adjuvant chemotherapy was associated with better survival of patients aged $\leq 70$ years with the 21-gene RS 26-30. Interestingly, we found that breast cancer-specific and overall survival rates in patients with the RS 26-30 and who received chemotherapy were higher in younger versus older patients and those with grade III versus grade I-II tumor. Additional clinical parameters such as Ki-67 proliferative index or expression levels of hormone receptor, which represent tumor biology or subtype, may provide additional information to aid a treatment decision for this subgroup of breast cancer patients $[14,15]$.

Since the introduction of multigene assays, use of the 21-gene RS test in clinical practice has gradually increased and is anticipated to be widely utilized as a treatment decision aid in ER-positive and HER2-negative early-stage breast cancer patients [16, 17]. However,

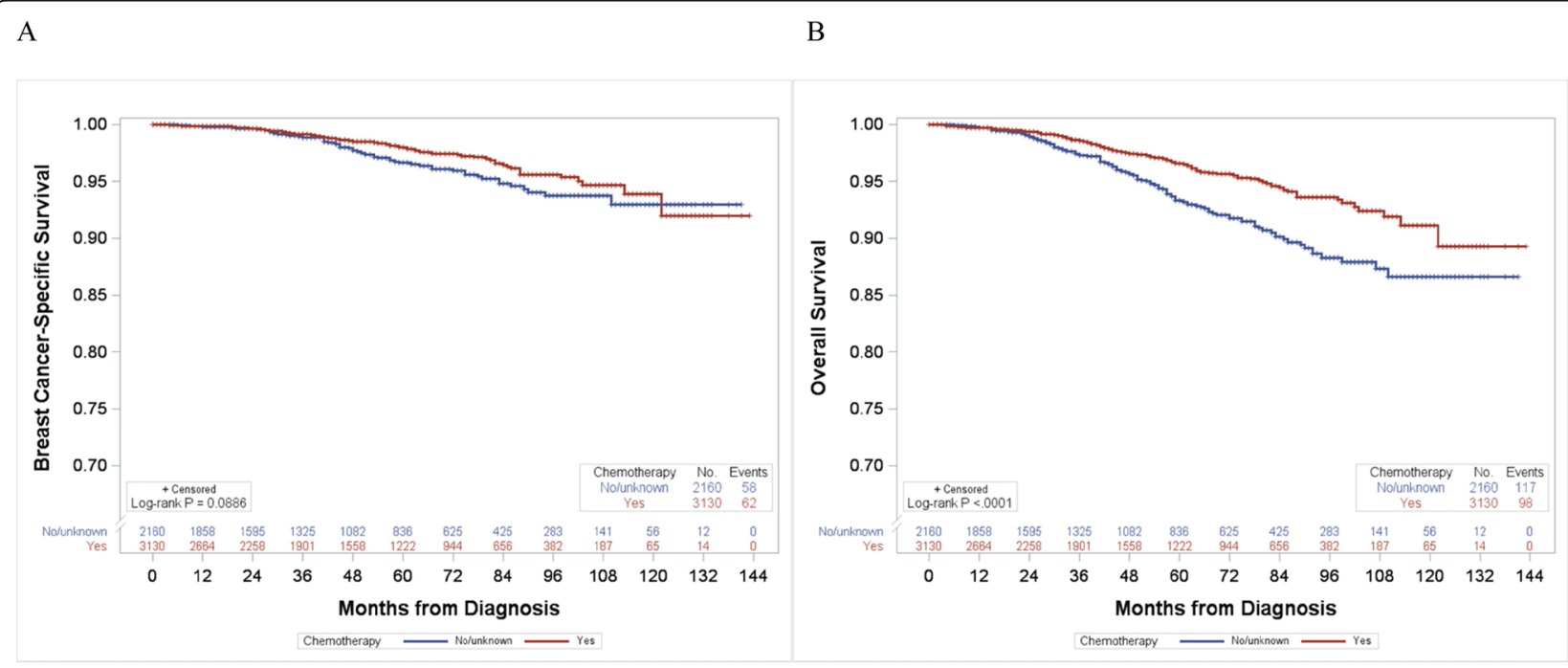

Fig. 3 Breast cancer-specific and overall survival curves according to chemotherapy use in patients aged $\leq 70$ years old with the RS $26-30$ in the SEER 2004-2015 dataset $(n=5290)$. a Breast cancer-specific survival and b overall survival 


$$
\text { A }
$$

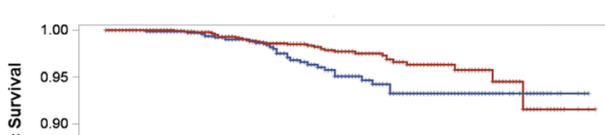

号 0.90

苛 0.85

这 0.80

营 0.75

0.70

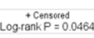

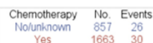

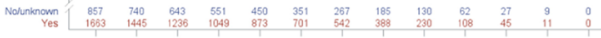

$\begin{array}{lllllllllllll}0 & 12 & 24 & 36 & 48 & 60 & 72 & 84 & 96 & 108 & 120 & 132 & 144\end{array}$ Months from Diagnosis

$\mathrm{C}$
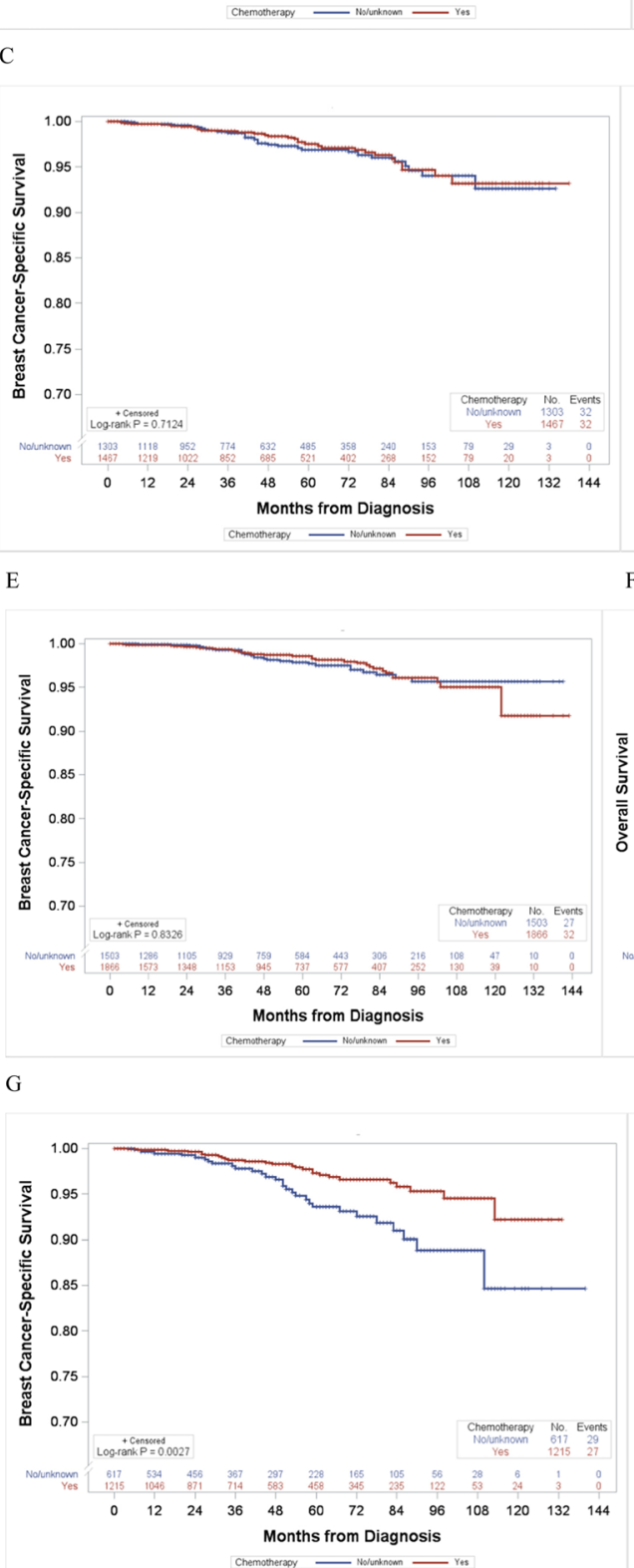

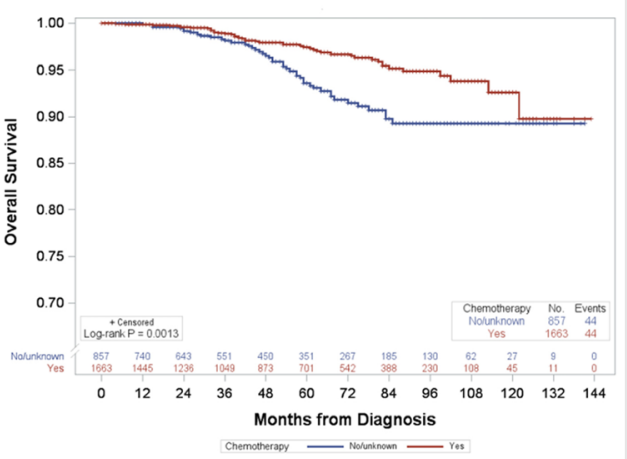

D
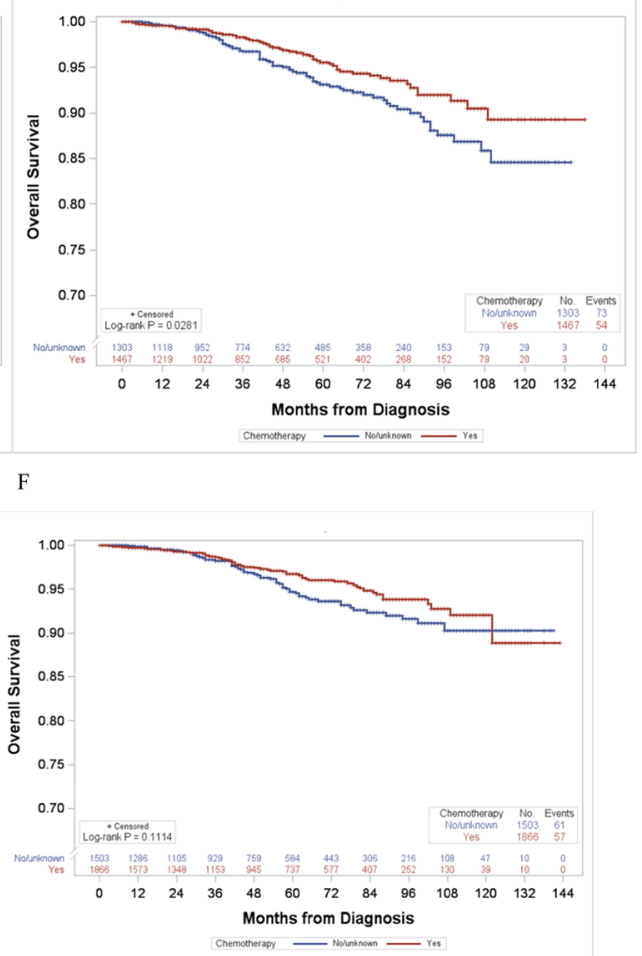

$\mathrm{H}$

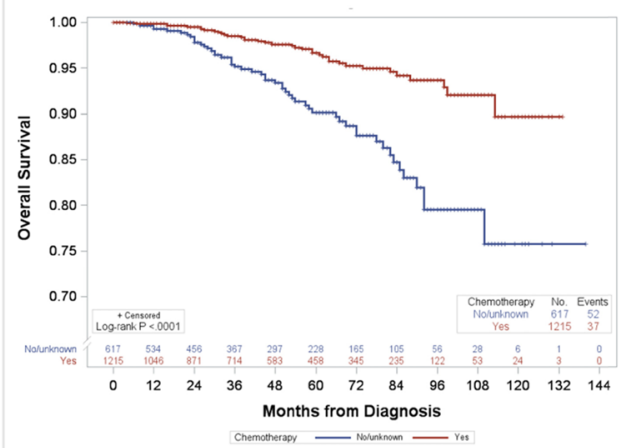

Fig. 4 (See legend on next page.) 
(See figure on previous page.)

Fig. 4 Breast cancer-specific and overall survival curves according to chemotherapy use among patients with age $\leq 70$ years and the RS 26-30, stratified by age and tumor grade. $\mathbf{a}$ Breast cancer-specific survival in patients $\leq 56$ years of age, $\mathbf{b}$ overall survival in patients $\leq 56$ years of age, $\mathbf{c}$ breast cancer-specific survival in patients 57-70 years of age, $\mathbf{d}$ overall survival in patients 57-70 years of age, e breast cancer-specific survival in patients with grade I-II tumor, $\mathbf{f}$ overall survival in patients with grade I-II tumor, $\mathbf{g}$ breast cancer-specific survival in patients with grade III tumor, and $\mathbf{h}$ overall survival in patients with grade III tumor

clinicians should be aware that the 21-gene RS is a relative numeric calculated, not an absolute parameter for predicting patient prognosis. In previous clinical trials that validated the 21 -gene RS assay $[2,3]$, the prevalence of patients with the RS $18-30$ classified as an intermediate-risk group was 21-22\%. However, the prevalence of breast cancer with the RS 18-30 was higher (33\%) in a nation-wide cancer database in the USA [9]. The prevalence of the intermediate RS group varied across countries: from $20.0 \%$ in the Japanese to $40.7 \%$ in the Israeli with early-stage breast cancer $[7,18,19]$. Among breast cancer patients with intermediate risk of the RS 18-30, approximately one out of five patients had the RS of 26-30 in our study using the SEER and the previous studies [7, 9].
Similarly to previous studies [7,9], we observed that the intermediate-risk group, RS 18-30, was heterogeneous in their demographic and clinicopathological characteristics. Compared to patients with the RS 18-25, breast cancer patients with the RS 26-30 were more likely to have aggressive tumor: larger size, higher grade, and single hormone receptor positivity. Also, we found that a $5 \%$ increased risk of overall mortality per 1 unit increase in the RS among breast cancer patients with the RS 18-30, which was consistent with a previous finding [9]. Breast cancer-specific mortality was increased by $9 \%$ per 1 unit increase in the RS among our study population with the RS 18-30.

As expected [20], chemotherapy-treated patients had more aggressive clinicopathological features. However,

Table 3 Hazard ratios (HR) for breast cancer-specific and overall mortality by chemotherapy use in patients aged $\leq 70$ years old with the RS 26-30

\begin{tabular}{|c|c|c|c|c|}
\hline & \multicolumn{2}{|l|}{ Breast cancer-specific mortality } & \multicolumn{2}{|l|}{ Overall mortality } \\
\hline & $\begin{array}{l}\text { No/unknown chemotherapy } \\
\text { (Reference) }\end{array}$ & $\begin{array}{l}\text { Yes chemotherapy } \\
{[\mathrm{HR}(95 \% \mathrm{Cl})]}\end{array}$ & $\begin{array}{l}\text { No/unknown chemotherapy } \\
\text { (Reference) }\end{array}$ & $\begin{array}{l}\text { Yes chemotherapy } \\
{[\mathrm{HR}(95 \% \mathrm{Cl})]}\end{array}$ \\
\hline \multicolumn{5}{|l|}{ 2004-2015 dataset $(n=5290)$} \\
\hline No. of events & 58 & 62 & 117 & 98 \\
\hline Age-adjusted model & 1.00 & 0.76 (0.53 to 1.09$)$ & 1.00 & 0.63 (0.48 to 0.83 ) \\
\hline $\begin{array}{l}\text { Age- and clinicopathological factor-adjusted } \\
\text { model }\end{array}$ & 1.00 & $0.66(0.45$ to 0.95$)$ & 1.00 & 0.56 (0.42 to 0.74$)$ \\
\hline $\begin{array}{l}\text { Age- and clinicopathological and treatment } \\
\text { factor-adjusted model }\end{array}$ & 1.00 & 0.68 (0.47 to 0.99$)$ & 1.00 & 0.58 (0.44 to 0.76$)$ \\
\hline \multicolumn{5}{|l|}{ 2010-2015 dataset $(n=3540)$} \\
\hline No. of events & 22 & 23 & 40 & 40 \\
\hline Age-adjusted model & 1.00 & 0.73 (0.40 to 1.32 ) & 1.00 & 0.75 (0.48 to 1.18$)$ \\
\hline $\begin{array}{l}\text { Age- and clinicopathological factor-adjusted } \\
\text { model }\end{array}$ & 1.00 & 0.63 (0.34 to 1.15$)$ & 1.00 & 0.67 (0.43 to 1.05$)$ \\
\hline $\begin{array}{l}\text { Age- and clinicopathological and treatment } \\
\text { factor-adjusted model }\end{array}$ & 1.00 & 0.64 (0.35 to 1.18$)$ & 1.00 & 0.72 (0.45 to 1.14$)$ \\
\hline \multicolumn{5}{|l|}{ 2004-2009 dataset $(n=1750)$} \\
\hline No. of events & 36 & 39 & 77 & 58 \\
\hline Age-adjusted model & 1.00 & 0.77 (0.49 to 1.23$)$ & 1.00 & 0.56 (0.40 to 0.79 ) \\
\hline $\begin{array}{l}\text { Age- and clinicopathological factor-adjusted } \\
\text { model }\end{array}$ & 1.00 & 0.68 (0.43 to 1.09$)$ & 1.00 & 0.50 (0.35 to 0.71$)$ \\
\hline $\begin{array}{l}\text { Age- and clinicopathological and treatment } \\
\text { factor-adjusted model }\end{array}$ & 1.00 & 0.71 (0.44 to 1.13) & 1.00 & 0.51 (0.36 to 0.72 ) \\
\hline
\end{tabular}

Age at diagnosis is used by categorization into $\leq 50,51-60$, and 61-70 years. Adjusted clinicopathological factors are year of diagnosis (2004-2005, 2006-2007, 2008-2009 in 2004-2009 dataset and 2010-2011, 2012-2013, and 2014-2015 in 2010-2015 dataset), race/ethnicity (White, Black, and other), history of cancer (no and yes), marital status (married and single/other), histologic type (ductal, lobular, and mixed ductal-lobular/other), tumor stage (T1b, T1C, and T2-3), grade (I, II, III, and missing), and ER/PR status (both ER/PR-positive and either ER/PR-positive). Treatment factors are type of surgery (breast-conservation surgery and mastectomy) and radiation therapy (no/unknown and yes)

$R S$ recurrence score, $H R$ hazard ratio, $\mathrm{Cl}$ confidence interval, No. number 
after adjusting for demographic and clinicopathological factors in our study, chemotherapy was significantly associated with a lower risk of breast cancer-specific and overall mortality in patients $\leq 70$ years old with the 21 gene RS 26-30. This suggested that adjuvant chemotherapy might be beneficial to patients who are $\leq 70$ years old and have the RS 26-30 even though they are classified as having an intermediate risk of recurrence. The study by Ibraheem et al. [9] also reported that chemotherapy was related to a $32 \%$ lower risk of overall mortality in patients with node-negative breast cancer and the 21-gene RS 26-30. In a re-analysis of the National Surgical Adjuvant Breast and Bowel Project (NSABP) B20 trial excluding presumed HER2-positive cases, significantly better distant recurrence-free survival rates were observed in patients with the 21 -gene $\mathrm{RS} \geq 26$ who received chemotherapy [4].

Our study was one of the largest studies examining the effect of chemotherapy on survival in breast cancer patients with an intermediate risk for recurrence. The SEER data which capture $>98 \%$ of incident cancer cases in 18 geographical regions provide a large number of breast cancer cases with survival outcome and the 21gene RS [11, 21]. In addition, we selected our patient population according to the NCCN practice guidelines and focused analyses on patients with the RS 26-30, which will help decision-making for chemotherapy in patients with the RS 26-30. Our study also has several limitations mostly related to inherent limitations in the SEER database: it did not provide information on disease recurrence, endocrine therapy use, and details of chemotherapeutic regimens. Also, receipt of chemotherapy and radiation therapy was under-reported in the SEER program compared to the Medicare claims data restricted to patients aged $\geq 65$ years [22]. Given that systemic chemotherapy tends to be administered to patients with better performance status which could not be assessed in this study, we cannot rule out the possibility of residual confounding by unmeasured factors in our analyses. In addition, although the distribution of the 21-gene RS did not differ between 2010 and 2015 and 2004-2009 dataset, about $5 \%$ of total breast cancer cases had HER2-positive, borderline, or undetermined status in 2010-2015 dataset just before the final exclusion step. Given that 1with HER2-positive were more likely to have the higher 21-gene RS, analyses of 2004-2009 data may have additional confounding due to lack of HER2 status. Nevertheless, when we performed sensitivity analyses including patients with HER2 positive, borderline, or unknown status in 2010-2015 data, our results did not change. Another limitation was the lack of information on menopausal status. Thus, we could not examine premenopausal and postmenopausal breast cancer, separately.

\section{Conclusions}

Our findings suggest that adjuvant chemotherapy provides a significant survival benefit to patients with an intermediate risk for recurrence, particularly those with the 21-gene RS 26-30, and particularly for younger patients and those with high-grade tumors. Given that the 21-gene RS is a promising tool that can guide a chemotherapy decision in early-stage breast cancer patients with hormone receptor-positive and HER2-negative tumor, a shared-decision making with patients, using the multigene assay result and discussions about known risks and benefits of chemotherapy should be warranted in the era of personalized medicine.

\section{Additional files}

\begin{abstract}
Additional file 1: Figure with CONSORT diagram. Abbreviation: RS, recurrence score; SEER, Surveillance, Epidemiology, and End Results; ER, estrogen receptor; PR, progesterone receptor; HER2, human epidermal growth factor receptor 2. (DOCX $115 \mathrm{~kb}$ )
\end{abstract}

Additional file 2: Figure with distribution of the 21-gene RS. Blue color represents the 2004-2009 dataset, green, 2010-2015 dataset just before the final step of excluding HER2-positive, borderline, or unknown status, and red, the final analytic 2010-2015 dataset remaining only HER2-negative category after exclusion of all criteria. Percentage of the 21-gene RS is calculated in each dataset. Dotted line shows a cutoff used in this study. The mean of the 21-gene RS among datasets was not significantly different ( $t$-test, $P=0.296$ between the 2004-2009 dataset and 2010-2015 dataset before excluding HER2; $P=0.065$ between 2004 and 2009 dataset and the final 2010-2015 dataset after excluding HER2; and $P=0.305$ between 2010 and 2015 dataset before excluding HER2 and the final 2010-2015 dataset after excluding HER2). (DOCX 80 kb)

Additional file 3: Table with multivariate analysis for risks of breast cancer-specific and overall mortality by the 21-gene RS in sensitivity analyses that included breast cancer with HER2-positive, borderline, and unknown status in 2010-2015 ( $n=20,943)$. (DOCX $15 \mathrm{~kb}$ ) (DOCX $14 \mathrm{~kb}$ )

Additional file 4: Table with patients' characteristics by chemotherapy use in patients aged $\leq 70$ years old with the recurrence score (RS) 26-30. *Other race includes American Indian, Alaska Native, and Asian or Pacific Islander. †Other marital status includes separated, divorced, and widowed categories. Abbreviation: RS, recurrence score; No., number; ER, estrogen receptor; PR, progesterone receptor. (DOCX 18 kb)

\section{Abbreviations \\ Cl: Confidence interval; ER: Estrogen receptor; HER2: Human epidermal growth factor receptor 2; HR: Hazard ratio; ICD-O-3: International Classification of Diseases for Oncology, 3rd Edition; NCCN: National Comprehensive Cancer Network; NCDB: National Cancer Data Base; NSABP: National Surgical Adjuvant Breast and Bowel Project; \\ PR: Progesterone receptor; RS: Recurrence score; TAlLORx: Trial Assigning Individualized Options for Treatment}

\section{Acknowledgements}

Not applicable.

\section{Authors' contributions}

SP and YP were responsible for the conception and design of the study. SP, YH, and $Y L$ collected and analyzed the data. All authors interpreted the data and wrote the manuscript. All authors read and approved the final manuscript.
Funding

Not applicable. 


\section{Availability of data and materials}

The data that support the findings of this study are available from the SEER program (https://seer.cancer.gov/), but restrictions apply to the availability of these data, which were used under permission for the current study, and so are not publicly available. Data are however available from the authors upon reasonable request and with permission of the SEER.

\section{Ethics approval and consent to participate}

Not applicable.

\section{Consent for publication}

Not applicable.

\section{Competing interests}

The authors declare that they have no competing interests.

\section{Author details}

'Division of Public Health Sciences, Department of Surgery, Washington University School of Medicine, 660 South Euclid Avenue, Campus Box 8100, St. Louis, MO 63110, USA. ${ }^{2}$ Division of Breast Surgery, Department of Surgery, Yonsei University College of Medicine, Seoul, Republic of Korea. ${ }^{3}$ Department of Breast Surgery, First Hospital of China Medical University, Shenyang, China. ${ }^{4}$ Division of Medical Oncology, Department of Medicine, Washington University School of Medicine, St. Louis, MO, USA.

Received: 16 May 2019 Accepted: 28 August 2019

\section{Published online: 16 October 2019}

\section{References}

1. Early Breast Cancer Trialists' Collaborative Group (EBCTCG). Effects of chemotherapy and hormonal therapy for early breast cancer on recurrence and 15-year survival: an overview of the randomised trials. Lancet. 2005; 365(9472):1687-717.

2. Paik S, Shak S, Tang G, Kim C, Baker J, Cronin M, et al. A multigene assay to predict recurrence of tamoxifen-treated, node-negative breast cancer. $\mathrm{N}$ Engl J Med. 2004;351(27):2817-26.

3. Paik S, Tang G, Shak S, Kim C, Baker J, Kim W, et al. Gene expression and benefit of chemotherapy in women with node-negative, estrogen receptorpositive breast cancer. J Clin Oncol. 2006;24(23):3726-34.

4. Geyer CE Jr, Tang G, Mamounas EP, Rastogi P, Paik S, Shak S, et al. 21-gene assay as predictor of chemotherapy benefit in HER2-negative breast cancer. NPJ Breast Cancer. 2018;4:37.

5. Sparano JA, Gray RJ, Makower DF, Pritchard Kl, Albain KS, Hayes DF, et al. Prospective validation of a 21-gene expression assay in breast cancer. $\mathrm{N}$ Engl J Med. 2015;373(21):2005-14.

6. Sparano JA, Gray RJ, Makower DF, Pritchard KI, Albain KS, Hayes DF, et al. Adjuvant chemotherapy guided by a 21-gene expression assay in breast cancer. N Engl J Med. 2018;379(2):111-21.

7. Stemmer SM, Steiner M, Rizel S, Soussan-Gutman L, Ben-Baruch N, BareketSamish A, et al. Clinical outcomes in patients with node-negative breast cancer treated based on the recurrence score results: evidence from a large prospectively designed registry. NPJ Breast Cancer. 2017;3:33.

8. Gluz O, Nitz UA, Christgen M, Kates RE, Shak S, Clemens M, et al. West German Study Group Phase III PlanB Trial: first prospective outcome data for the 21-gene recurrence score assay and concordance of prognostic markers by central and local pathology assessment. J Clin Oncol. 2016;34(20):2341-9.

9. Ibraheem AF, Press DJ, Olopade OI, Huo D. Community clinical practice patterns and mortality in patients with intermediate oncotype DX recurrence scores: who benefits from chemotherapy? Cancer. 2019;125(2): 213-22.

10. NCCN Clinical Practice Guidelines in Oncology (NCCN Guidelines): Breast Cancer; Version 3.2018. https://www.nccn.org/professionals/physician_gls/ default.aspx. Accessed 10 Dec 2018.

11. Petkov VI, Miller DP, Howlader N, Gliner N, Howe W, Schussler N, et al. Breast-cancer-specific mortality in patients treated based on the 21-gene assay: a SEER population-based study. NPJ Breast Cancer. 2016;2:16017.

12. Johnson CJ, Weir HK, Fink AK, German RR, Finch JL, Rycroft RK, et al. The impact of National Death Index linkages on population-based cancer survival rates in the United States. Cancer Epidemiol. 2013;37(1):20-8.

13. Sparano JA. TAlLORx: trial assigning individualized options for treatment (Rx). Clin Breast Cancer. 2006;7(4):347-50.
14. Curigliano G, Burstein HJ, E PW, Gnant M, Dubsky P, Loibl S, et al. Deescalating and escalating treatments for early-stage breast cancer: the St. Gallen International Expert Consensus Conference on the Primary Therapy of Early Breast Cancer 2017. Ann Oncol. 2017;28(8):1700-12.

15. Nitz U, Gluz O, Christgen M, Kates RE, Clemens M, Malter W, et al. Reducing chemotherapy use in clinically high-risk, genomically low-risk pN0 and pN1 early breast cancer patients: five-year data from the prospective, randomised phase 3 West German Study Group (WSG) PlanB trial. Breast Cancer Res Treat. 2017;165(3):573-83.

16. Jasem J, Amini A, Rabinovitch R, Borges VF, Elias A, Fisher CM, et al. 21-gene recurrence score assay as a predictor of adjuvant chemotherapy administration for early-stage breast cancer: an analysis of use, therapeutic implications, and disparity profile. J Clin Oncol. 2016;34(17):1995-2002.

17. Williams AD, Reyes SA, Arlow RL, Tchou J, De La Cruz LM. Is age trumping genetic profiling in clinical practice? Relationship of chemotherapy recommendation and Oncotype DX recurrence score in patients aged $<50$ years versus $\geq 50$ years, and trends over time. Ann Surg Oncol. 2018;25(10): 2875-83.

18. Toi M, Iwata H, Yamanaka T, Masuda N, Ohno S, Nakamura S, et al. Clinical significance of the 21-gene signature (Oncotype DX) in hormone receptorpositive early stage primary breast cancer in the Japanese population. Cancer. 2010;116(13):3112-8.

19. Lee MH, Han W, Lee JE, Kim KS, Park H, Kim J, et al. The clinical impact of 21-gene recurrence score on treatment decisions for patients with hormone receptor-positive early breast cancer in Korea. Cancer Res Treat. 2015;47(2):208-14

20. Kozick Z, Hashmi A, Dove J, Hunsinger M, Arora T, Wild J, et al. Disparities in compliance with the Oncotype DX breast cancer test in the United States: a National Cancer Data Base assessment. Am J Surg. 2018;215(4):686-92.

21. Noone A, Howlader N, Krapcho M, Miller D, Brest A, Yu M, et al: SEER Cancer Statistics Review, 1975-2015, National Cancer Institute. Bethesda, MD, https://seer.cancer.gov/csr/1975_2015/, based on November 2017 SEER data submission, posted to the SEER web site, April 2018.

22. Noone AM, Lund JL, Mariotto A, Cronin K, McNeel T, Deapen D, et al. Comparison of SEER treatment data with Medicare claims. Med Care. 2016; 54(9):e55-64.

\section{Publisher's Note}

Springer Nature remains neutral with regard to jurisdictional claims in published maps and institutional affiliations. 